\title{
Power Consumption Monitoring System using IOT
}

\author{
Bharathi R. \\ Associate Professor \\ BMSIT \& M, Dept. of CSE
}

\author{
Madhushree M. E. \\ BE, Dept. of CSE \\ BMSIT\&M
}

\author{
Priyanka Kumari \\ BE, Dept. of CSE \\ BMSIT\&M
}

\begin{abstract}
Lack of resources established in the present world is initiating everyone towards energy efficient technologies. Among all these resources, power is one which needs to be monitored and controlled as per the need since electricity consumption is increasing day-by day. We live in a world where almost everything runs on electricity. $67 \%$ of their sources used to produce electricity are non -renewable sources of energy. Power is the soul of world which is related to the electricity and "electricity" is the word which now rules the world. So, proper utilization of these resources is of immense important to us. Though many technological innovations are taking place in this world, existing electricity consumption billing process seems in India to be very old fashioned and does not meet the latest technology available. In this paper we present a newly designed digital meter based on a very cheap distributed components like microcontroller architecture and current sensors.
\end{abstract}

The power lines which already exist and connect every household in a particular area as it does not require any new installation or erection for establishment of communication channels. So the system doesn't require placing other cables and along with this we are using WIFI to communicate with the servers and users. By measuring current and voltage, we can analyze energy consumption, make the world smarter place and make better decisions using Internet of Things.

\section{Keywords}

Internet of things (IOT), Smart electric meter reading, Microcontroller, Current sensors

\section{INTRODUCTION}

Though electricity is very essential in day to day life, the proper utilization of it must be done. We can properly consume the electricity as well as calculate the electricity consumption by using the electric meter. The vulnerability about the supply of energy can tell the working of whole economy, especially in creating financial aspects. It is the necessity to manage consumption of electricity due to limited availability of resources. So the aim of this paper is to recognize and eliminate the misuse of electricity.

Internet of things has helped many organizational systems to improve efficiency, increase the speed of processes, minimize error and prevent theft by coding and tracking the objects. Computing and communications has its future in the technological transformation brought by the IOT. Power consumption can be reduced to a great extent if we can monitor our daily power usage and switch off appliances which are unnecessary consuming electricity. This paper focuses on developing a monitoring system using the concept of Internet of Things.

\section{EXISTING SYSTEMS}

In existing system either an electronic energy meter or an electro-mechanical meter is fixed in the premise for measuring the usage. The meters currently in use are only capable of recording KWH units. The KWH units used then still have to be recorded by meter readers monthly, on foot. The recorded data need to be processed by a meter reading company. For processing the meter reading, company needs to firstly link each recorded power usage data to an account holder and then determine the amount owned by means of the specific tariff in use many systems built on various platforms have been proposed by different research groups all over the world for Automatic Meter Reading. Tele watt meters were implemented to transmit data on monthly basis to a remote central office through a dedicated telephone line and a pair of modems.

A microprocessor or DSP- based meter is used in this to measure the electricity consumption of multiple users in a residential area. A master PC at the control center was used to send commands to a remote meter, which in turn transmitted data back, using the Power Line Communication technique. These techniques were mainly implemented in areas that had a fixed telephone network. Bluetooth energy meters were designed and implemented in some areas where several meters in close proximity, communicated wirelessly with a Master PC. In this measurement technique that encompasses the GSM network as a mean of transmitting energy data is more relevant. The GSM network offers most coverage in most developed and developing countries.

\section{PROPOSED SYSTEM}

We are developing an application where the application can be installed in computer or mobile. There will be a server where it will store the information about the usage of electricity and the amount will be calculated and added and stored in the server database. From this server the information is exchanged between the devices like mobile and computers. Every day the user can access the day to day information required about the amount of electricity utilized and the cost estimation from the server. So, from this the user can get alert of the power consumed and tries to reduce the usage of power by this automatically we can reduce the cost and save the money and it also helps in more usage of resources for further.

Smart Meter is used to monitor the power consumption and displays total power consumed and it is sent to server periodically. Server is used to store the data of individual homes for future analysis and also we can access data through mobile app and by PC through web page.

\section{METHODOLOGY}

The overall process of the paper is shown in the following figure. The hardware and the software interface are connected to each other to monitor the power consumption of the user and further, this will be monitored by server and will be uploaded to cloud from where the user can $\log$ on to the webpage in computer and App installed in the mobile. The smart meter will display the voltage, current and power on 16X2 LCD display. 


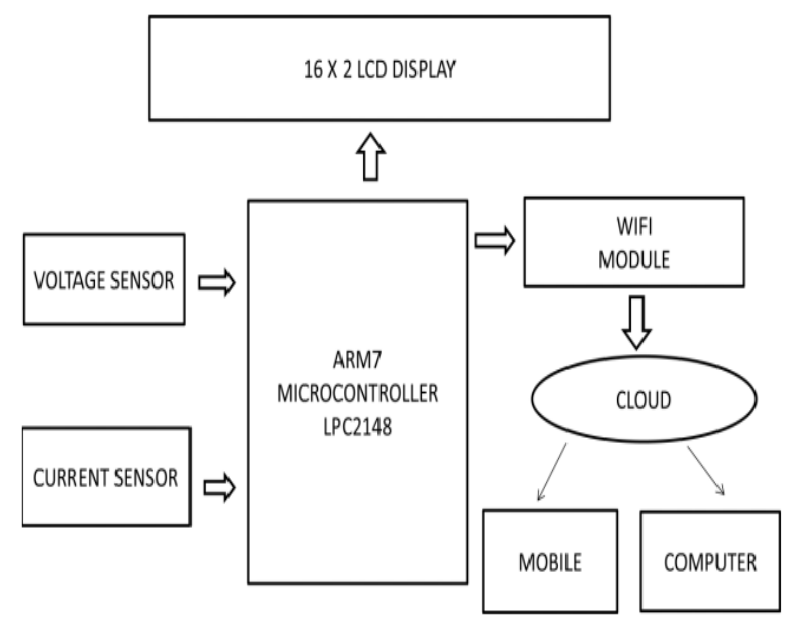

Fig. 1 Architectural Design of Meter

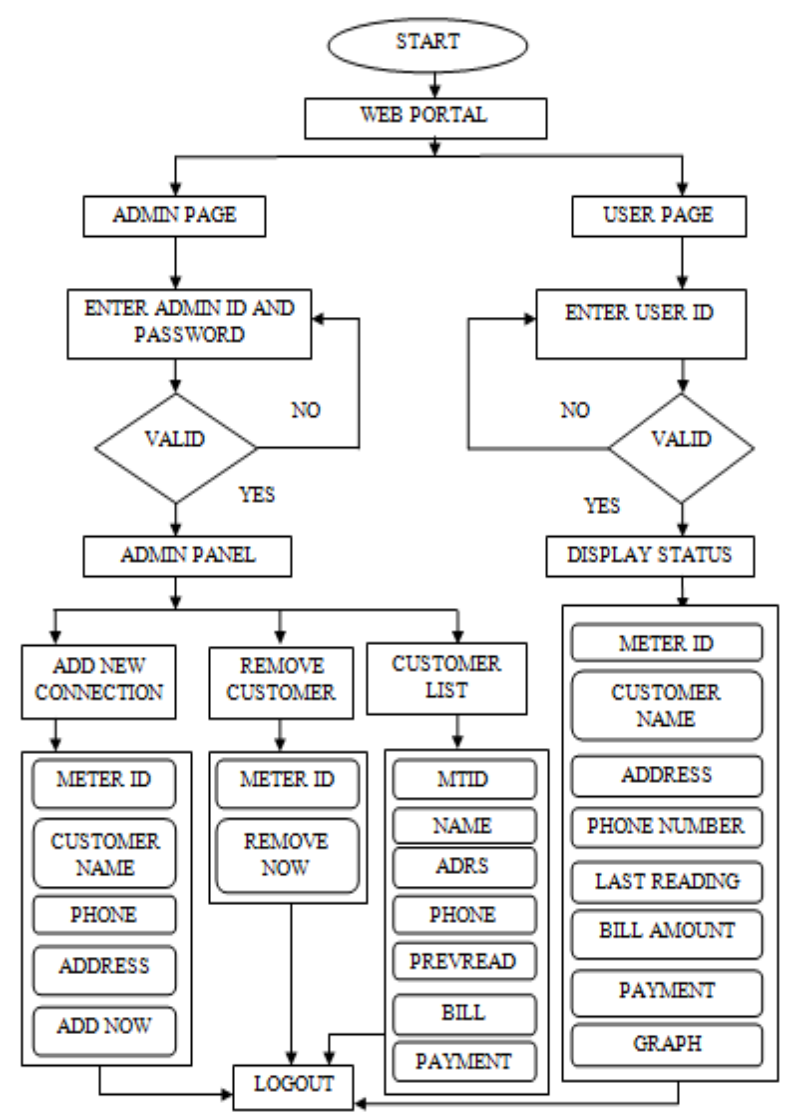

Fig 2: Activity Diagram

Activity diagram is another important diagram in UML to describe the dynamic aspects of the system. Activity diagram is basically a flowchart to represent the flow from one activity to another activity. The activity can be described as an operation of the system. So the control flow is drawn from one operation to another. This flow can be sequential, branched, or concurrent. Activity diagrams deal with all type of flow control by using different elements such as fork, join, etc.

The purpose can be described as:

- Draw the activity flow of a system.

- Describe the sequence from one activity to another.

- Describe the parallel, branched and concurrent flow of the system.

\section{IMPLEMENTATION}

Implementation consists of software and hardware implementation. In order to implement the website, we used MICRO KEIL VERSION 4, XAMPP, HTML, EMBEDDED C, JavaScript and PHP.Whereas to implement the hardware, we use voltage sensor, current sensor, LCD, ARM7 Microcontroller LPC2148, WI-FI Module, Web portal, Computer or Mobile. Current Sensing however poses much more difficult problems due to the rich harmonic content in the current waveform. Current transducer sensor not only requires a much wider measurement dynamic range, but also necessary to handling of a much wider frequency range.

Voltage Sensing is usually obtained by using either the voltage division method or a step down voltage transformer. Decision making about which method should be used, is related to the work necessities.

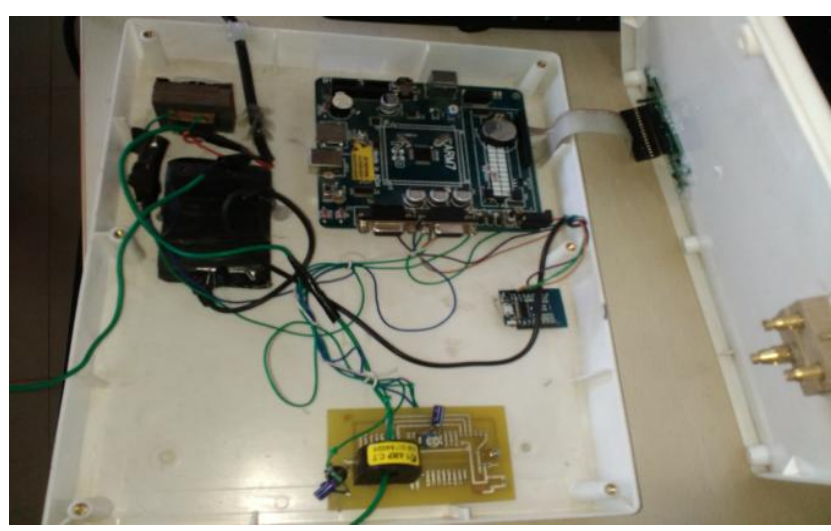

ARM7 MICROCONTROLLER LPC2148 is widely used IC from ARM-7 family and it is pre-loaded with many inbuilt peripherals making it more efficient and a reliable option for the beginners as well as high end application developer.

Wi-Fi compatible devices can connect to the Internet via a WLAN. A Web portal is most often a specially designed website that brings information together from diverse sources in a uniform way.

\section{FUTURE WORK}

- Issues in Smart Meter Data Analytics

To achieve metering intelligence as described in the previous sections, a number of technical issues need to be successfully addressed. The ability to work with very large volumes of data will be a key requirement. It is also essential that technologies be able to

Work with a variety of data such as weather information and consumer information, geographic data thus requiring techniques for efficient data fusion and integration.

\section{- Smart Meters and Big Data}

"Big data" is a term that is currently being used widely with data analytics. Big data has many interpretations but there are three key features that are highlighted: volume, velocity, and variance. Data captured by smart meters clearly relate to all these features thus satisfying the definition for big data. For example, moving from one meter reading per month to smart meter readings every $30 \mathrm{~min}$ results in a massive volume of data to manage. The data are collected in frequent time periods, and if technology is available for near real time analysis, many advantages could be achieved. 


\section{CONCLUSION}

The 21st century has brought great discoveries and advancements in the field of technology. These advancements also brought many challenges and require approaches to handle these challenges. Smart metering system is one such approach. This paper has presented a comprehensive survey of smart metering and electricity smart meter data analytics. Although there has been much opposition to electric meters due to privacy concerns, it is obvious that smart meters are here to stay and will be a "way of life" in the future.

The complete working model of a smart energy meter was built which uses existing WIFI Module system. The model satisfactorily worked with a Bulb. Automatic meter reading can be explained well using this system. Financial losses of electricity board can be minimized. Labour charges and effort can be reduced. The error, time delay that occurs due to manual metering can be avoided to a great extent. Electrical line fault detection has been made easy for the electricity board. Finally but not the least this type of meter supports remote metering which is the future of energy meters.

- Unique Id for Admin and User.

- Only Admin can modify any data and add new users.

- Power used by the users can be monitored hour/daily manner.

\section{REFERENCES}

[1] Lun Cuifen, Zhang Xiaoqin, Li Yanping, Liuce "The Electric Meter Reading System Based on Wireless Micro Computer" - 2010 (IEEE Paper)
[2] ChristianBeckel and SilviaSantini, "Improving device level electricity consumption breakdowns in private households using ON/OFF events," ACM SIGBED Rev., vol. 9, pp. 32-38, 2012.

[3] Garrab, A.; Bouallegue, A.; Ben Abdallah, "A new AMR approach for energy saving in Smart Grids using Smart Meter and partial Power Line Communication", IEEE First International Conference on Renewable Energies and Vehicular Technology (REVET), pp. $263-269$, march 2012

[4] Marco Casini , "Internet of things for Energy efficiency of buildings," International Scientific Journal Architecture and Engineering. - 2013

[5] Shu-ping Le, Hong Zeng, Jian Qiu, Song Zhang "Design and Implementation of Wireless Power Monitoring System for Public Buildings" - 2013 (IEEE Paper)

[6] S. Lanzisera and D. Pajak, "Communicating Power Supplies: Bringing the Internet to the Ubiquitous Energy Gateways of Electronic Devices," IEEE Internet Of Things Journal, Vol. 1, No. 2, pp. 153-160, April 2014.

[7] S.Madakam and S.Tripathi, "Internet of Things (IoT): A Literature Review," Journal of Computer and Communications, pp.164-173, May 2015

[8] V.Preethi, G. Harish Design and Implementation of Smart Energy Meter 2015 (IEEE Paper) 